\title{
A Multichannel Integrated Circuit for Electrical Recording of Neural Activity, With Independent Channel Programmability
}

\author{
Carolina Mora Lopez, Student Member, IEEE, Dimiter Prodanov, Dries Braeken, Ivan Gligorijevic, \\ Wolfgang Eberle, Senior Member, IEEE, Carmen Bartic, Robert Puers, Fellow, IEEE, and \\ Georges Gielen, Fellow, IEEE
}

\begin{abstract}
Since a few decades, micro-fabricated neural probes are being used, together with microelectronic interfaces, to get more insight in the activity of neuronal networks. The need for higher temporal and spatial recording resolutions imposes new challenges on the design of integrated neural interfaces with respect to power consumption, data handling and versatility. In this paper, we present an integrated acquisition system for in vitro and in vivo recording of neural activity. The ASIC consists of 16 low-noise, fully-differential input channels with independent programmability of its amplification (from 100 to $6000 \mathrm{~V} / \mathrm{V}$ ) and filtering (1-6000 $\mathrm{Hz}$ range) capabilities. Each channel is AC-coupled and implements a fourth-order band-pass filter in order to steeply attenuate out-of-band noise and DC input offsets. The system achieves an input-referred noise density of $37 \mathrm{nV} / \sqrt{\mathrm{Hz}}$, a NEF of 5.1, a CMRR $>60 \mathrm{~dB}$, a THD $<1 \%$ and a sampling rate of $30 \mathrm{kS} / \mathrm{s}$ per channel, while consuming a maximum of 70 $\mu \mathrm{A}$ per channel from a single $3.3 \mathrm{~V}$. The ASIC was implemented in a $0.35 \mu \mathrm{m}$ CMOS technology and has a total area of $5.6 \times 4.5$ $\mathrm{mm}^{2}$. The recording system was successfully validated in in vitro and in vivo experiments, achieving simultaneous multichannel recordings of cell activity with satisfactory signal-to-noise ratios.
\end{abstract}

Index Terms-CMOS interface, in vitro recording, in vivo recording, low-noise neural recording, multichannel integrated circuit, programmable biosensor interface.

\section{INTRODUCTION}

$\mathbf{T}$ HE investigation of neural networks is not only of great importance to understand the basic function of the brain, but moreover it can lead to a better comprehension of severe brain disorders such as Alzheimer's and Parkinson's diseases, epilepsy, autism and psychiatric disorders [1], [2]. Novel advances in computational capabilities, design tools and microelectrode fabrication techniques allow for the development of neural prostheses capable of interfacing with single neurons and neuronal networks. To obtain this resolution of interfacing, neural probes and the electrodes fabricated on these devices

Manuscript received July 29, 2011; revised October 18, 2011; accepted December 10, 2011. This work was supported in part by the Flemish Government (IWT SBO Grant BrainStar). This paper was recommended by Associate Editor M. Stanacevic.

C. Mora Lopez, D. Prodanov, D. Braeken, and W. Eberle are with Imec, B-3001 Leuven, Belgium (e-mail: moralope@imec.be).

I. Gligorijevic, R. Puers, and G. Gielen are with the Electrical Engineering Department-ESAT, Katholieke Universiteit Leuven, B-3001 Leuven, Belgium.

C. Bartic is with the Department of Physics and Astronomy, Katholieke Universiteit Leuven, B-3001 Leuven, Belgium.

Digital Object Identifier 10.1109/TBCAS.2011.2181842 need to be customized to the anatomy and morphology of the recording site [3]. Besides this, multi-site recording functionality that allows for simultaneous recording of neural activity in different brain areas is a requisite for neural interface devices.

Currently, multi-electrode arrays are being combined with CMOS electronics in an effort to convert classical passive neural probes into more sophisticated active neural probes. This migration, mainly motivated by the need of proximity between electrodes and circuitry, and the increasing density in multielectrode arrays are generating important circuit design challenges with regard to miniaturization, noise and power dissipation reduction, programmability and handling of large quantity of output data.

Neural signals, usually measured with extracellular microelectrodes, consist of action potentials (APs) or spikes superimposed on low-frequency local field potentials (LFPs). Both APs and LFPs are important to answer neuroscientific questions and they can be, to some extent, studied distinctly using band-separation techniques. APs are estimated by examining the power variation of the signals in the high-frequency range (typically 300-6000 Hz), whereas the LFPs are assessed by the power variation in the low-frequency range (i.e., 1-200 Hz) [4]. In addition, the spikes of a small group of neurons near the electrode can be detected and classified, providing information on single-unit activity. LFPs are usually divided into bands in order to study activity patterns of a larger population of neurons, giving information about the state of the neural network which is difficult to capture using spiking activity from only a few neurons [5]. Recently, it has been shown that when spikes and LFPs are measured simultaneously, substantially more information about an external stimulus can be extracted when analyzing firing and phase information together [6], [7]. Thus, simultaneous examination of both spiking activity and LFPs can provide a better understanding of the organization of the synaptic inputs (e.g., LFPs) to neurons and of the relationship between spikes and LFPs [8]. One of the major challenges for the recording of neural signals is therefore the successful and simultaneous conditioning of both AP and LFP signals with optimized parameters according to their nature. As these two kinds of signals have different amplitude ranges $(30-1000 \mu \mathrm{V}$ for APs and $1-10 \mathrm{mV}$ for LFPs) and bandwidths, they also require different gains, filters and even electrode sizes in order to be optimally measured. 


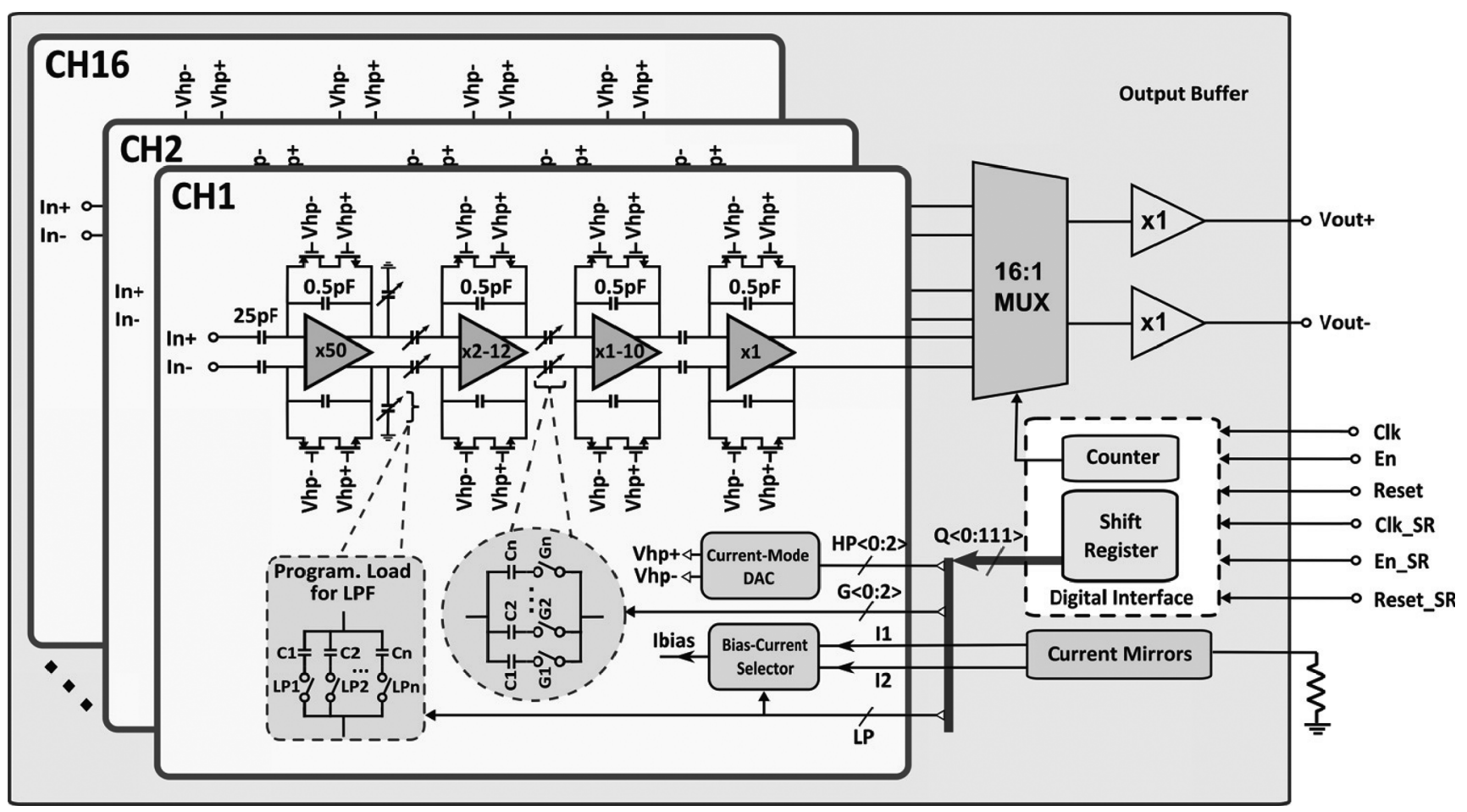

Fig. 1. Architecture of the 16-channel neural recording system.

Existing neural recording architectures include some or all of the following components: pre-amplification, filtering, analog multiplexing, additional amplification, buffering, digitalization and wired/wireless data transmission/storage [9]-[18]. More sophisticated features such as spike detection circuits [14], [19], or LFP measurement circuits [20] are also included in some designs in order to obtain data reduction in multi-electrode applications. Discrete [9], [10], hybrid [11] and custom integrated [12]-[18] neural recording implementations in the last three decades have attempted to address problems such as electrodecircuitry interconnection, noise/interference, size, power consumption and data transmission. However, because of (i) the constantly increasing density of multi-electrode arrays, (ii) the need for fully implantable recording devices and (iii) the use of smaller electrodes, several of these problems still remain. A complete review of neural interfaces is presented in [21].

In this paper we propose a 16-channel low-noise neural recording system that addresses the programmability of the amplifiers and filters in order to accurately monitor neural APs and LFPs. This architecture (Section II) offers individual channel programmability for simultaneous conditioning of different neural signals and electrode characteristics. We demonstrate successful performance characterization of the fabricated circuit (in Section III-A) and also successful in vitro and in vivo recordings with satisfactory signal-to-noise ratios (in Sections III-B and III-C).

\section{Proposed Architecture And Circuit Design}

The architecture of the proposed neural recording system is shown in Fig. 1. It consists of 16 AC-coupled fully-differential input channels, a time-division analog multiplexer and an output buffer. Each channel provides fourth-order band-pass filtering implemented in four amplification stages, so that highfrequency noise/interferences and large input DC-offsets can be rejected. Programmable gains from 100 to $6000 \mathrm{~V} / \mathrm{V}$ are achieved by digitally selectable ( 3 bits) capacitor arrays placed in the second and third amplification stages. Also, the cutoff frequencies of the high-pass filter and low-pass filter can be digitally programmed with 3 and 1 bits, respectively. The programming of the channels is done through a shift register interface (pins Clk_SR,En_SR and Reset_SR in Fig. 1), which has 112 registers in total (7 registers per channel).

The system offers two operating modes (selectable by software): first, simultaneous recording from the 16 recording channels or, secondly, recording from only one specific channel. In the second operation mode, the channels that are not recording are switched off in order to save power.

\section{A. Preamplifier}

As neural recording involves the measurements of very small voltages in the $\mu \mathrm{V}$-range, noise can become a limiting factor in the performance of the system. The total noise at the input of the neural interface is composed of the noise introduced by the electrodes (and the interface with the brain tissue) and the input-referred noise of the electronic circuitry. The former is determined by the material and impedance of the electrodes, as well as other characteristics of the electrode-electrolyte/tissue interface. Several studies have been carried out to estimate or model the electrode noise [23]-[25]. It has been found that for $\mathrm{Pt}$, for example, the total source noise from the electrodes and the tissue can be estimated from the measured total impedance, by calculating the thermal noise associated with the real (resistive) part of that impedance [24]. Therefore, thermal noise levels 
are highly dependent on the impedance of the electrode-tissue interface for each individual recording site (due to the foreign body reaction) and the selected recording bandwidth [25]. The input-referred noise of the electronic circuitry mainly includes thermal and flicker noise of the circuit input stage, which must be kept lower than the electrode noise so that it has a minor contribution to the overall noise. Therefore, the design of the preamplifier involves numerous trade-offs with other important specifications such as power consumption and area. If the input stage is an operational transconductance amplifier (OTA), the ideal input-referred noise, assuming differential-pair transistors in the sub-threshold region and a first-order frequency response, can be expressed as [26]

$$
V_{n i, r m s}=\sqrt{\frac{4 k T \cdot U_{T} \cdot}{\kappa^{2} \cdot I_{D}} \cdot \frac{\pi}{2} \cdot B W}
$$

where $k$ is Boltzmann's constant, $T$ is the absolute temperature, $U_{T}$ is the thermal voltage, $\kappa$ is the sub-threshold gate coupling coefficient, $I_{D}$ is the nominal current in each input transistor and $B W$ is the $-3 \mathrm{~dB}$ bandwidth of the amplifier. As can be seen, for a given bandwidth the noise is inversely proportional to the square root of the supply current, that is, there exists a trade-off between noise and power consumption.

An important figure of merit in the design of biopotential amplifiers is the noise-efficiency factor (NEF) [27], which scales the noise, power and bandwidth of a design against a reference BJT amplifier. The NEF is calculated as

$$
N E F=V_{n i, r m s} \sqrt{\frac{2 I_{\text {tot }}}{4 k T \cdot U_{T} \cdot \pi \cdot B W}}
$$

where $I_{\text {tot }}$ is the total supply current of the amplifier.

In this design, the preamplifier [Fig. 2(a)] is a fully-differential folded-cascode OTA with a closed-loop gain of 50, set by a feedback capacitor ratio of $25 \mathrm{pF} / 0.5 \mathrm{pF}$. The input differential pair is formed by two PMOS transistors operating in the sub-threshold (weak inversion) region, which maximizes their transconductance-over-drain-current ratio and, therefore, minimizes their thermal noise contribution [28]. Also, transistors M4-M5 and M10-M11 are operated in the strong inversion region to minimize their noise contribution, resulting in a trade-off with the output signal swing. All transistors are made as large as possible to minimize the flicker noise. Table I shows the dimensions and the operating conditions (drain currents and the inversion coefficients) of each transistor in the OTA. Simulation results show that the OTA has an open-loop gain of $105 \mathrm{~dB}$, a phase margin of $84^{\circ}$, a unit gain bandwidth of $760 \mathrm{kHz}$ and an input-referred noise of $1.9 \mu \mathrm{V}_{r m s}$, while consuming a total current of $20 \mu \mathrm{A}$. The inputs are capacitively coupled in order to eliminate DC offsets coming from the electrode-electrolyte/ tissue interface. A continuos-time common-mode feedback circuit [Fig. 2(b) [22]] has been used to control the common-mode voltage at the output nodes. Additional programmable gains of maximum 12 and $10 \mathrm{~V} / \mathrm{V}$ are added in the subsequent two stages, respectively.

\section{B. Filters}

The high-pass filtering characteristic in each channel is achieved by the parallel combination of the feedback capacitors

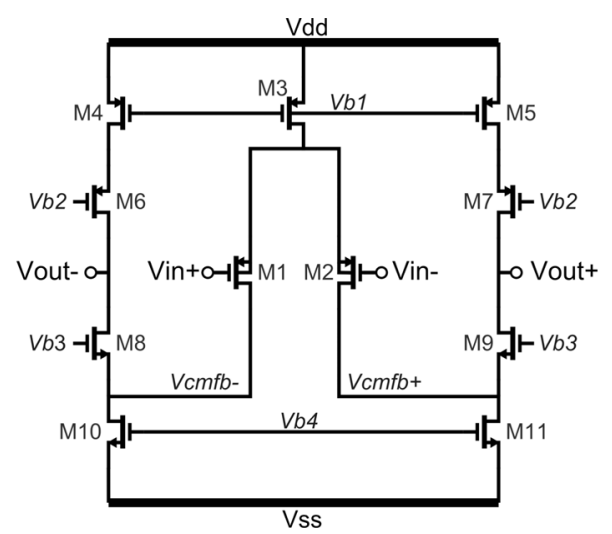

(a)

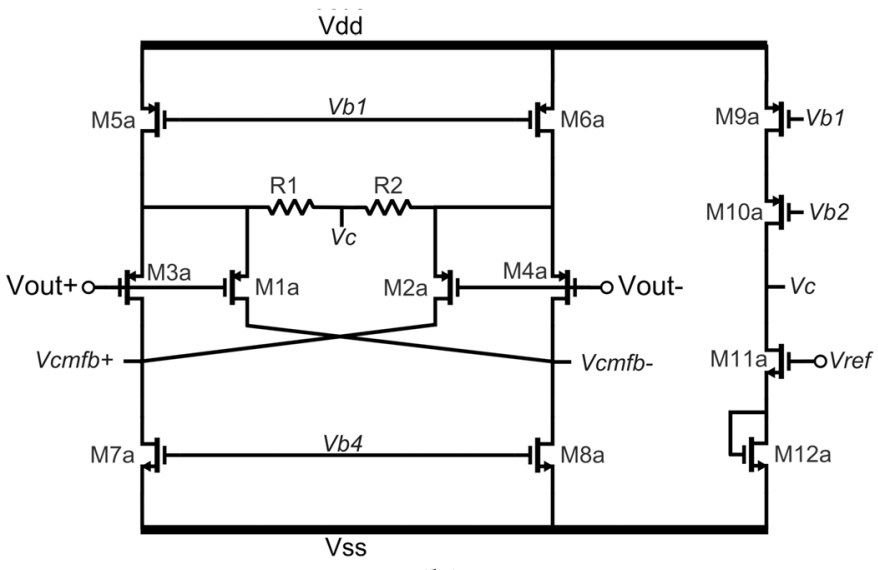

(b)

Fig. 2. Schematic of the preamplifier. (a) Fully-differential folded-cascode input amplifier. (b) Continuos-time CMFB circuit [22].

TABLE I

Sizes AND OPERATING POINTS FOR TRANSISTORS IN THE PREAMPLIFIER

\begin{tabular}{lccc}
\hline \hline Devices & $\boldsymbol{W / L}(\mu \mathbf{m})$ & $\boldsymbol{I}_{D}(\mu \boldsymbol{A})$ & Inversion Coefficient \\
\hline M1, M2 & $500 / 4$ & 8 & 0.67 \\
M3 & $60 / 50$ & 16 & 140.8 \\
M4, M5 & $7.5 / 50$ & 2 & 138.9 \\
M6, M7 & $16.25 / 1$ & 2 & 1.3 \\
M8, M9 & $6.5 / 1$ & 2 & 1.3 \\
M10, M11 & $30 / 160$ & 10 & 227.3 \\
\hline
\end{tabular}

and the feedback PMOS-NMOS pseudo-resistor elements, which are biased in the deep-subthreshold region. These voltage-controlled pseudo-resistors [28] can achieve very high resistance values in the order of $10^{12} \Omega$, providing an area-efficient way to implement very low frequency filters. The cutoff frequency can be tuned via a current-mode digital-to-analog converter (Fig. 3) that changes the gate voltages of the transistors (i.e., the resistance), in order to accept or reject the LFP signal frequencies. This circuit consists of a wide-swing cascode current mirror that copies the selected current to the diode-connected transistors $M n$ and $M p$, which are sized with large $W / L$ ratios. A similar method was previously described by Yin et al. [30]. The appropriate biasing of the gate of the PMOS and NMOS transistors enables the linearization of the 


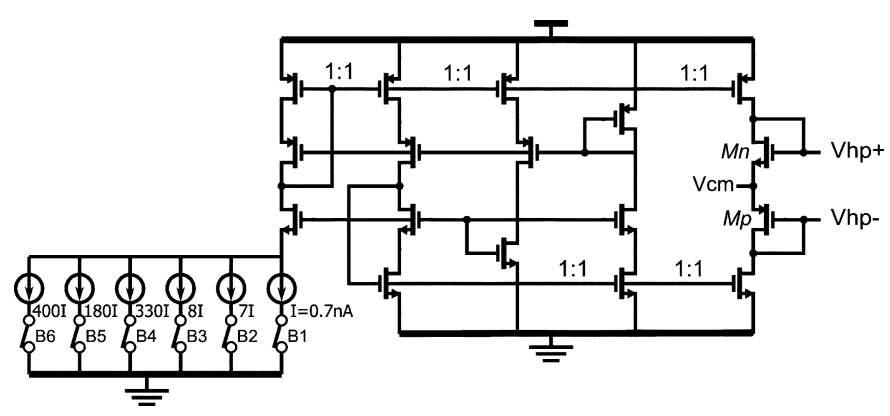

Fig. 3. Current-mode digital-to-analog converter to control the PMOS-NMOS pseudo-resistors.

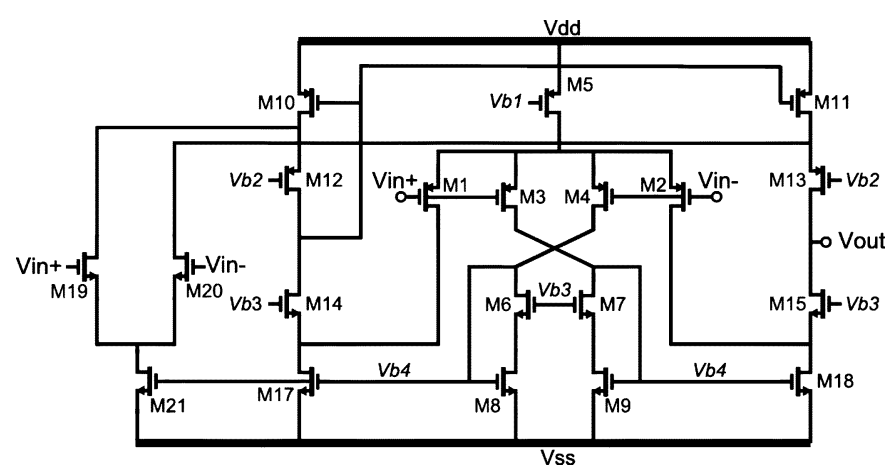

Fig. 4. Rail-to-rail output buffer to drive an external load (ADC). It uses the recycling folded-cascode architecture described in [29].

resistance, as well as the programming of process-independent cutoff frequency values. A total of 6 predefined cutoff frequencies can be digitally selected with 3 control bits.

The fourth-order low-pass filter characteristic is implemented by the cascade of four first-order voltage integrators, consisting of an OTA and a load capacitor. The load capacitors are implemented as programmable capacitor arrays that allow the selection (1 bit) of two different frequency ranges: one for the LFP signals and another for the AP signals. Also, when the low-frequency LFP-recording mode is selected, the supply current of the amplifiers is lowered in order to save power $(\sim 50 \%)$. In total, 7 bits are used to program independently the gain and the band-pass filter of each channel.

\section{Output Stage}

The output stage consists of an analog time-division multiplexer and an output buffer. For the implementation of the time-division analog multiplexer, an array of standard transmission gates is used to achieve rail-to-rail voltage operation. The multiplexer is controlled by a digital counter in order to sample each channel with a minimum rate of $12.5 \mathrm{kS} / \mathrm{s}$. Two single-ended output buffers are placed at the output of the multiplexer to drive an external load (an ADC or other instrument) of maximum $70 \mathrm{pF}$. The design of this buffer is also critical as it has to be able to handle a large input voltage swing and a minimum sampling rate of $200 \mathrm{kS} / \mathrm{s}$.

The schematic of the output buffer is shown in Fig. 4. It is a rail-to-rail amplifier, designed to have a settling time $<1 \mu \mathrm{s}$ (i.e., for a maximum sampling rate of $60 \mathrm{kS} / \mathrm{s}$ per channel)

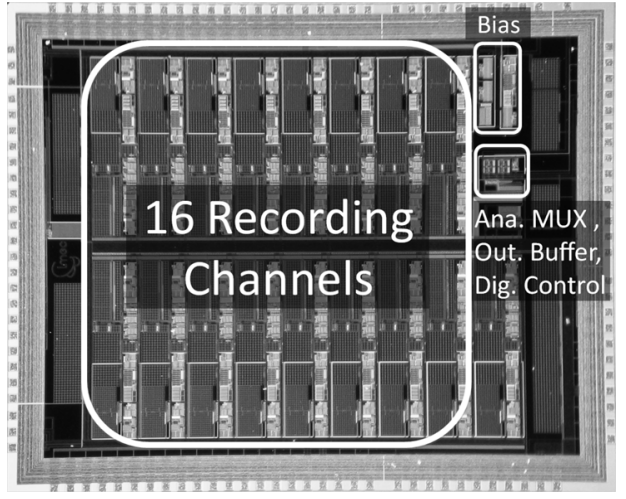

Fig. 5. Die photo of the 16-channel neural recording system. The total area is $5.6 \times 4.5 \mathrm{~mm}^{2}$ and the core area is $4.1 \times 3.8 \mathrm{~mm}^{2}$.

with a current consumption of $360 \mu \mathrm{A}$. The architecture of this amplifier is based on the recycling folded-cascode architecture described by Assaad et al. [29], which achieves enhanced transconductance, gain and slew rate while consuming the same power budget as a conventional folded-cascode amplifier. In our design, we have included double PMOS/NMOS differential pairs in order to achieve rail-to-rail input operation. The shown amplifier makes part of a two-stage Miller CMOS OTA, where the second stage is just one single transistor with active load. In this way we can also achieve rail-to-rail output operation.

\section{EXPERIMENTAL RESULTS}

The 16-channel neural recording system in Fig. 1 has been implemented and fabricated in a $0.35 \mu \mathrm{m}$ AMIS CMOS technology. The capacitors are implemented as metal-insulator-metal capacitors and the resistors as polysilicon resistors. The die (Fig. 5) occupies a core area of $4.1 \times 3.8 \mathrm{~mm}^{2}$ and a total area of $5.6 \times 4.5 \mathrm{~mm}^{2}$. The area of one channel is $0.76 \mathrm{~mm}^{2}$.

\section{A. Performance Measurement}

Table II summarizes the measured characteristics of the neural recording system and Table III gives an overview of the power consumption distribution in the different building blocks of the circuit. The chip consumes $1.8 \mathrm{~mA}$ from a $3.3 \mathrm{~V}$ supply (i.e., $5.9 \mathrm{~mW}$ ) when the chip is configured to record AP signals (200-6000 Hz bandwidth) and $0.8 \mathrm{~mA}$ (i.e., $2.6 \mathrm{~mW}$ ) when it records LFP signals (1-200 Hz bandwidth). One channel consumes $70 \mu \mathrm{A}$ and $17 \mu \mathrm{A}$ in the two configurations, respectively. Fig. 6 shows the frequency response of one channel measured with a spectrum analyzer. Gains of approximately 100, 200, 500, 1000, 2000, 3000, 4000 and $6000 \mathrm{~V} / \mathrm{V}$ can be digitally programmed for all the frequency ranges. The measured $-3-\mathrm{dB}$ programmable cutoff frequencies of the high-pass filter are 2.6, $5.6,13.4,213,323$ and $572 \mathrm{~Hz}$, and the programmable cutoff frequencies of the low-pass filter are $230 \mathrm{~Hz}$ and $6.2 \mathrm{kHz}$. The input-referred noise (Fig. 7) was also measured for the different programmable modes and it was calculated to be between 2.3 and $2.9 \mu \mathrm{V}_{r m s}$ (integrated from $1 \mathrm{~Hz}$ to $6 \mathrm{kHz}$ ). The CMRR, also measured with a spectrum analyzer, is shown 
This article has been accepted for inclusion in a future issue of this journal. Content is final as presented, with the exception of pagination.

TABLE II

MEASURED PERFormance CHARACTERISTICS

\begin{tabular}{|c|c|c|c|c|c|}
\hline \multirow{2}{*}{ Parameter } & \multicolumn{5}{|c|}{ Measured Value } \\
\hline & This Work & [15] & [16] & [17] & [18] \\
\hline No. of Channels & 16 & 16 & 128 & 256 & 32 \\
\hline Supply Volt. [V] & 3.3 & 1.8 & 3.3 & 3 & 3 \\
\hline Power/Channel* $[\mu \mathbf{W}]$ & $\begin{array}{l}11.5 \text { (LFP range) } \\
231 \text { (AP range) }\end{array}$ & 10.85 & 40.14 & 15 & 75 \\
\hline Input-ref. Noise $\left[\mu \mathbf{V}_{r m s}\right]$ & $2.3-2.9$ & 5.4 & 4.9 & 7 & 6.9 \\
\hline NEF & 5.1 & 4.9 & - & 4.6 & - \\
\hline CMRR/PSRR [dB] & $\begin{array}{l}>65 / 70(\text { LFP range }) \\
>63 / 70(\text { AP range })\end{array}$ & $-1-$ & $90 / 80$ & $-1-$ & $134 / 62.7$ \\
\hline Gain $[V / V]$ & $100-6000$ & 3000 & $700-1000$ & $250-2500$ & $2500 / 8000$ \\
\hline HP 3-dB Cutoff [Hz] & $2.6-572$ & 100 & $0.1-200$ & $0.01-70$ & $0.1-1000$ \\
\hline LP 3-dB Cutoff [kHz] & $0.23 / 6.2$ & 9.2 & $2-20$ & $0.5-5$ & 8 \\
\hline Filter Order (HP/LP) & $4^{t h} / 4^{t h}$ & $1^{s t} / 1^{s t}$ & $1^{s t} / 1^{s t}$ & $2^{n d} / 1^{s t}$ & $2^{n d} / 2^{n d}$ \\
\hline THD & $0.75 \%\left(15 \mathrm{mV}_{p p}\right.$ input $)$ & $0.67 \%\left(160 \mu \mathrm{V}_{p p}\right.$ input $)$ & - & $0.7 \%\left(4.4 \mathrm{mV}_{p p}\right.$ input $)$ & - \\
\hline
\end{tabular}

* Only the power consumption in the analog signal conditioning blocks (amplifiers and filters) of each recording channel was taken into account.

TABLE III

CURRENT CONSUMPTION OF THE BUILDING BLOCKS IN THE Two RECORDING MODES

\begin{tabular}{lcc}
\hline \hline Block & Current AP & Current LFP \\
\hline Preamplifier + CMFB & $23 \mu \mathrm{A}$ & $1.15 \mu \mathrm{A}$ \\
Second Gain Stage & $13 \mu \mathrm{A}$ & $0.65 \mu \mathrm{A}$ \\
Third Gain Stage & $13 \mu \mathrm{A}$ & $0.65 \mu \mathrm{A}$ \\
Fourth Stage - Buffer & $21 \mu \mathrm{A}$ & $1.05 \mu \mathrm{A}$ \\
Output Buffer & $360 \mu \mathrm{A}$ & $360 \mu \mathrm{A}$ \\
Biasing Circuits & $16 \mu \mathrm{A}$ & $16 \mu \mathrm{A}$ \\
Programming Circuits & $5.7 \mu \mathrm{A}$ & $5.7 \mu \mathrm{A}$ \\
Total Current Consumption & $1.8 \mathrm{~mA}$ & $0.8 \mathrm{~mA}$ \\
\hline
\end{tabular}
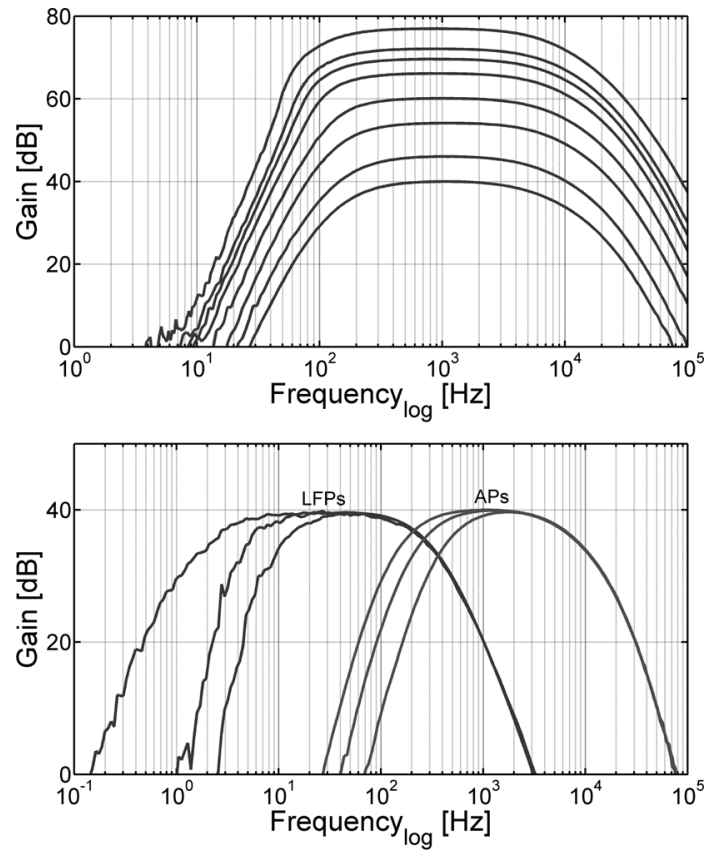

Fig. 6. Transfer function of one channel, measured for different bandwidths and gains.

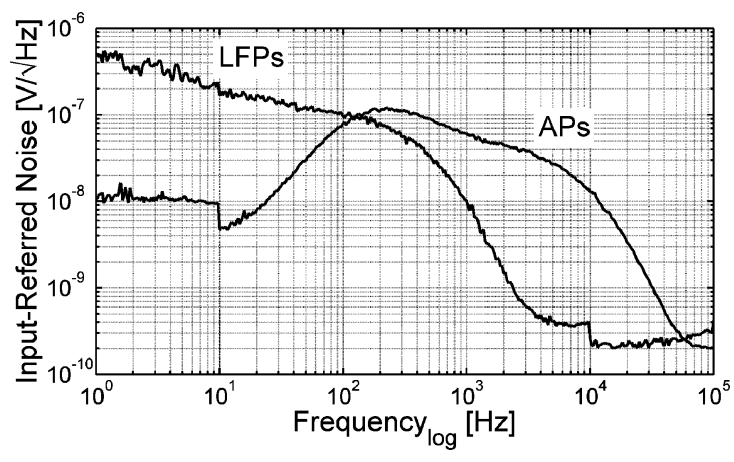

Fig. 7. Measured input-referred voltage noise density for one channel with grounded inputs and a gain of $6000 \mathrm{~V} / \mathrm{V}$.

in Fig. 8. Comparison with recently reported neural recording systems is also included in Table II. This work achieves a good noise level and a wide range of channel-independent programmable options (including very selective filters), while still keeping an acceptable NEF. Several design trade-offs (e.g., power/area/configurability, power/gain, power/area/noise, power/area/filter-order) were introduced in our design to achieve high configurability, linearity, filter-selectivity and low noise at the expense of power and area consumptions. Further design optimizations for power reduction could include the integration of the ADC on the same chip, thus avoiding the use of power-consuming output buffers.

\section{B. In Vitro Measurement}

In order to test the functionality of the recording system, we cultured primary cardiomyocytes on the chips. Action potentials from cardiac cells have the same frequency distribution as neural signals with slightly higher amplitudes, and they are used in our experiments because of the ease of the cell culturing, the spontaneous beating, and the straightforward interpretation of the data. To make in vitro cultures feasible, we packaged multi-electrode test chips on a custom PCB. A glass ring was glued on top of the PCB to contain the cells and the cell medium. 


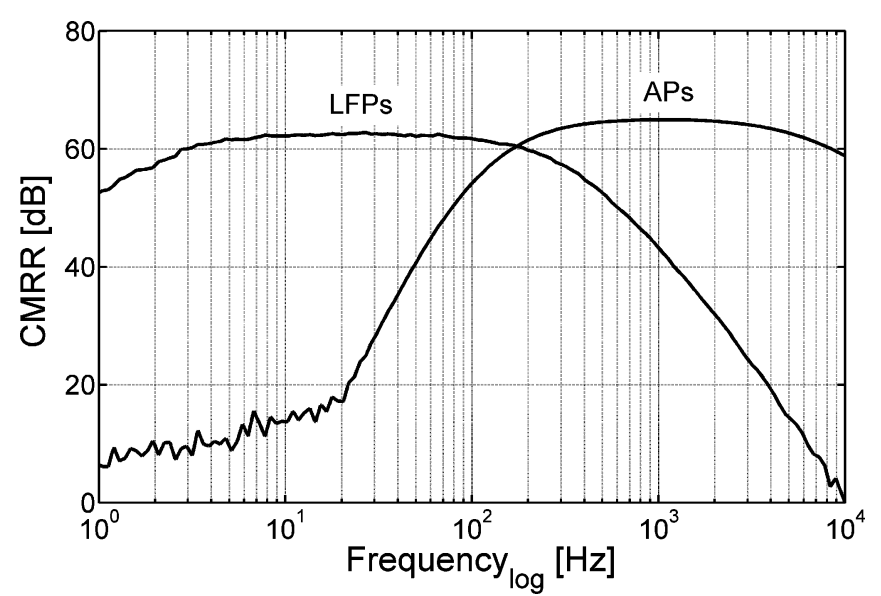

Fig. 8. Measured CMRR for one channel with shorted inputs and a gain of $6000 \mathrm{~V} / \mathrm{V}$.

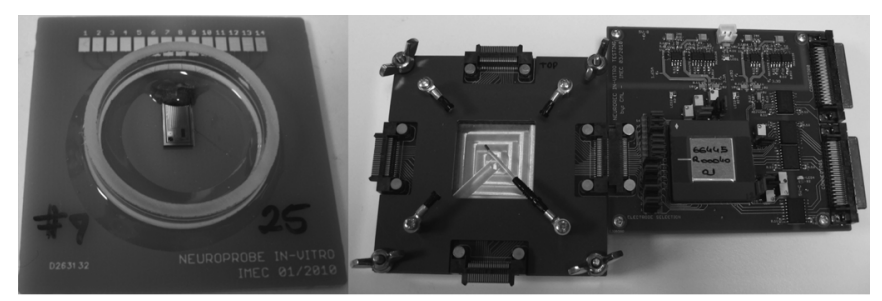

(a)

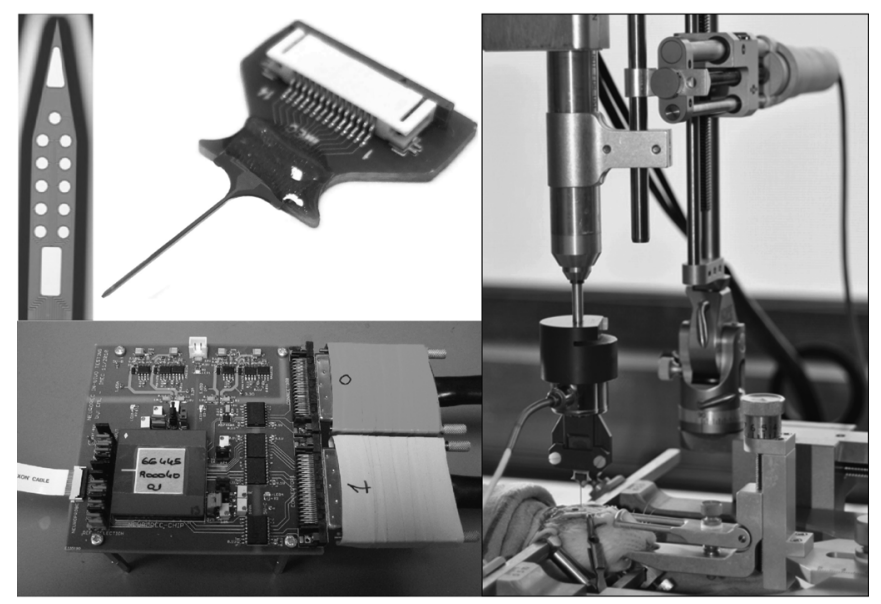

(b)

Fig. 9. Pictures of the setups used for neural recording. (a) In vitro setup consisting of a packaged multi-electrode (probe) test chip (left), a mechanical structure to contact the probe test chip and the PCB $\left(10 \times 9 \mathrm{~cm}^{2}\right)$ with the recording chip (right). (b) In vivo setup consisting of a 1-cm-long neural probe with 13 electrodes [31], the PCB $\left(9 \times 9 \mathrm{~cm}^{2}\right)$ with the recording chip (left) and a stereotactic frame connected to a micro-drive (right).

Cardiac cells were extracted from hearts of rat embryos and grown for 4 days in vitro on top of the packaged micro-fabricated probes with $50 \mu \mathrm{m}$ titanium-nitride electrodes [31]. The experimental setup [see Fig. 9(a)] consisted of a mechanical device to hold and allow electrical contact to the multi-electrode test chip, a PCB containing the recording chip and an external ADC with 12-bit resolution (Analog Devices AD7450A), and the interface with the computer (through a National Instruments
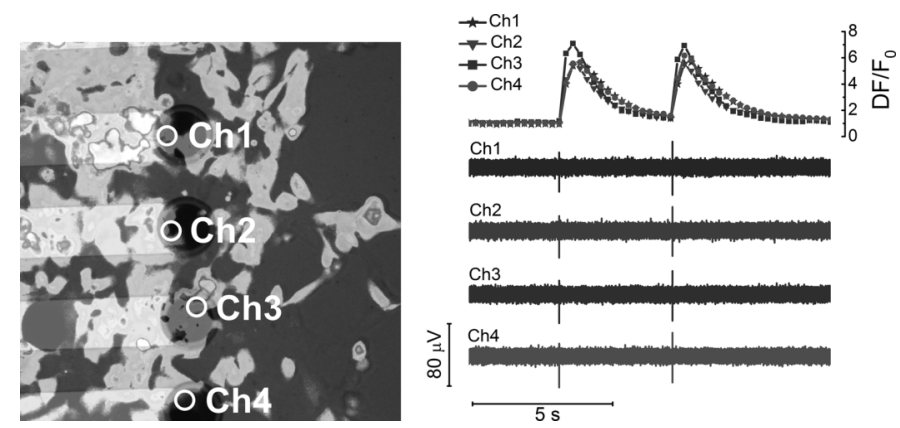

Fig. 10. Pseudo-fluorescence images of Fluo-4 loaded cardiac cells in overlay with neural probe images (left). Relative intracellular $\mathrm{Ca}^{2+}$ change $\left(\mathrm{DF} / \mathrm{F}_{0}\right.$ ) in the cell during spontaneous activity in the regions of interest $C h 1, C h 2, C h 3$ and $C h 4$ (top right). Recording of electrical action potentials (bottom right).

USB-6259 data acquisition card). The computer has custom-developed acquisition software (in Matlab), which allows the programming of the chip as well as the real-time transmission, display and storing of the recorded data. Signals with amplitudes as small as $40 \mu \mathrm{V}_{p-p}$ were detected due to the low input noise levels (around 10-30 $\mu \mathrm{V}_{p-p}$, coming mainly from the electrodes).

In order to validate the spikes that were recorded with our chips and to make sure they originate from cells on top of the electrodes, we performed Fluo4-AM calcium imaging experiments on the cardiac cells while recording electrical signals with the chip. This technique, based on the optical detection of changes in the intracellular calcium concentration $\left(\left[\mathrm{Ca}^{2+}\right]_{i}\right)$, allows to identify the origin of the electrical activity of the beating cells. Hence, large calcium transients in these embryonic cells are coinciding with action potentials, and thus the presence of a change in the $\left[\mathrm{Ca}^{2+}\right]_{i}$ on top of an electrode is represented by an action potential. This technique also allows us to measure the spatiotemporal distribution of the electrical signal on top of multiple electrodes. Fig. 10 represents the relative fluorescence intensity $\left(\mathrm{DF} / \mathrm{F}_{0}\right)$ at four different electrodes (areas of interest are marked with circles $C h 1, C h 2, C h 3$ and $C h 4$ ), captured by a CCD camera coupled to a fluorescence microscope. In this graph, we also show the electrical signals measured from four electrodes underneath the marked circles, which were measured with four adjacent channels in our recording system. It is clear that the measured APs coincide with large $\mathrm{Ca}^{2+}$ transients, although the latter last longer in comparison to the AP. The slow calcium transient is due to influx of $\mathrm{Ca}^{2+}$ inside the cell through opening of voltage-gated $\mathrm{Ca}^{2+}$ channels, and its relative slow clearance out of the cell by calcium transporters [32]. This experiment demonstrates that the signals recorded by the presented recording system and micro-fabricated chip correspond to actual cardiac action potentials.

\section{In Vivo Measurement}

We have also validated our integrated recording system in in vivo experiments. For that, we have used an implantable micro-machined 1-cm-long neural probe [31], containing $13 \mathrm{Pt}$ electrodes (one large triangular electrode with an area of $0.007 \mathrm{~mm}^{2}$, one large rectangular electrode with an area of $0.022 \mathrm{~mm}^{2}$ and 11 small circular electrodes with 10,25 or 


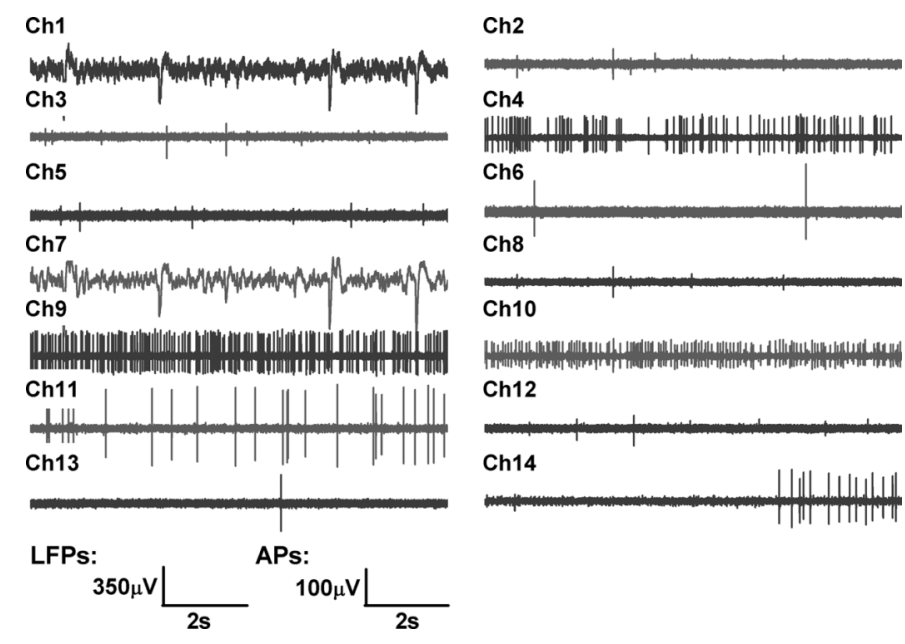

Fig. 11. In vivo simultaneous recordings (in rat) of LFPs and APs from 13 different Pt electrodes ( $25 \mu \mathrm{m}$ diameter) in a 1-cm-long neural probe positioned at a depth of $1.7 \mathrm{~mm}$. Channels $C h 1$ and $C h 7$ were configured to record LFPs (gain: $100 \mathrm{~V} / \mathrm{V}$, bandwidth: 1-200 Hz), while the other 12 channels were configured to record APs (gain: $1000 \mathrm{~V} / \mathrm{V}$, bandwidth: $300-6000 \mathrm{~Hz}$ ). Ch7 and Ch8 are connected to the same large electrode.

$50 \mu \mathrm{m}$ diameter). The neural probe was connected by means of a short flat cable to the custom PCB that holds the recording chip and the 12-bit external ADC (Analog Devices AD7450A). Similar to the in vitro setup, the PCB was connected to the computer through a National Instruments USB-6259 data acquisition card, and the real-time transmission, display and storing of the recorded data was done through custom-developed software in Matlab. A stereotactic frame with an external reference system was used to precisely control the implant position [Fig. 9(b)]. The insertion setup was shielded with a Faraday cage during experiments. With this setup, we were able to perform probe insertions and neural recording experiments in different brain regions of anesthetized rats (chloral hydrate $0.5 \mathrm{~g} / \mathrm{kg}$, s.c.; Sigma C-8383, Steinheim, Germany). All in vivo experiments were done in accordance with protocols approved by the local university animal ethics committee and the European Communities Council Directive.

All the recordings were performed in the motor cortex-hippocampus-thalamus insertion tract (coordinates: $2.2-3.3 \mathrm{~mm}$ posterior to Bregma, 1.7-2.2 $\mathrm{mm}$ lateral to the midline and up to $6 \mathrm{~mm}$ ventral to the dural surface). We have used three different types of neural probes (i.e., different electrode sizes: 10, 25 and $50 \mu \mathrm{m}$ diameter) with the same electrode arrangement, in order to evaluate the quality of the recordings under different input conditions (electrode impedance and noise). Spontaneous activity was recorded from the 13 contacts, using gains of $100 \mathrm{~V} / \mathrm{V}$ and $1000 \mathrm{~V} / \mathrm{V}$ for the LFP configuration (band-pass filter from $1 \mathrm{~Hz}$ to $200 \mathrm{~Hz}$ ) and the AP configuration (band-pass filter from $300 \mathrm{~Hz}$ to $6 \mathrm{kHz}$ ), respectively. Signals were sampled at $12.5 \mathrm{kS} / \mathrm{s}$ per channel (i.e., $200 \mathrm{kS} / \mathrm{s}$ total output data rate), digitized and transmitted in real time to the computer. Fig. 11 shows a 10 -sec extract from one of the recorded datasets from 14 different channels (i.e., $25 \mu \mathrm{m}$-diameter probe contacts at a depth of $1.7 \mathrm{~mm}$ ). In this recording, channels $C h 1$ and $C h 7$, which corresponded to the two large electrodes on the probe, were configured to sense LFPs; Ch8 is also connected to the large rect-
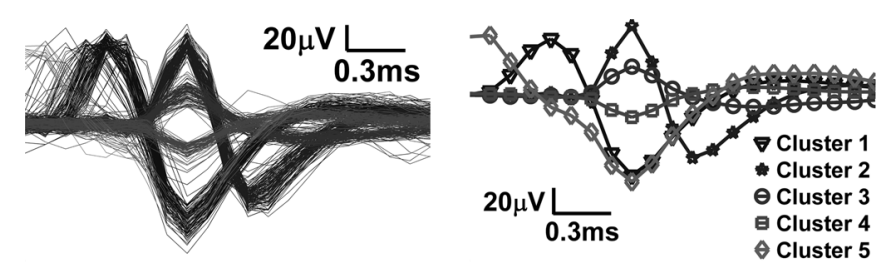

(a)
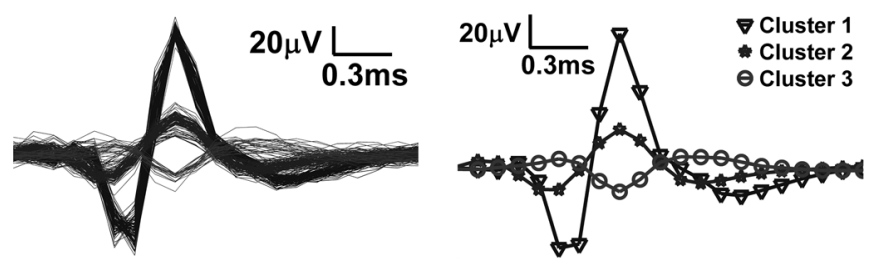

(b)
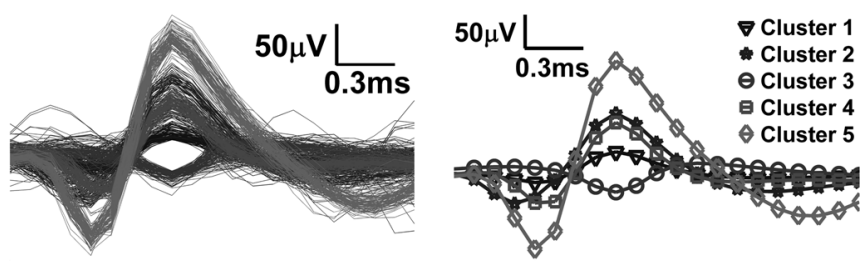

(c)

Fig. 12. Results of the spike sorting algorithm applied to datasets obtained in vivo with different neural probe types. Left: time-aligned raw neural spikes with several distinct neurons visible. Right: mean spike shapes per identified cluster. (a) Spikes measured with a $10 \mu \mathrm{m}$-diameter electrode. 5 different clusters were detected with $344,112,86,71$ and 11 spikes, respectively, and SNRs of 6.8, $5.8,2.1,1.6$ and 7.8 , respectively. (b) Spikes measured with a $25 \mu \mathrm{m}$-diameter electrode. 3 different clusters were detected with 1498, 118 and 26 spikes, respectively, and SNRs of 4.1, 1.3 and 0.7, respectively. (c) Spikes measured with a $50 \mu \mathrm{m}$-diameter electrode. 5 different clusters were detected with 1299, 366, 274, 113 and 169 spikes, respectively, and SNRs of 1.9, 5.8, 1.7, 4.6 and 12, respectively.

angular electrode, but it is configured to measure APs; the other channels, corresponding to the small electrodes, were configured to sense APs. The mean noise level was around $20 \mu \mathrm{V}_{p-p}$, with signal amplitudes up to $200 \mu \mathrm{V}_{p-p}$.

We have used Wave_Clus [33] to perform clustering of spontaneous APs recorded on each electrode of the neural probes. Wave_Clus is a Matlab toolbox that makes thresholding and sorting of spikes by using superparamagnetic clustering of the coefficients resulting from a wavelet transform of the spike waveforms [34]. In that way, spikes with similar shape, and that could eventually originate from a same neuron (i.e., unit), get separated and sorted in different clusters. Before thresholding, the algorithm performs extra data filtering in the range 300-3000 Hz. Fig. 12 shows examples of sorted spikes measured with one small electrode of each neural probe type. At the left, the figure shows time-aligned raw neural spikes with several distinct neurons visible. At the right, the mean spike shapes per identified cluster are shown. The SNR for each spike cluster was calculated as proposed in [35], i.e., the root-mean square (RMS) of a mean spike shape divided by the standard deviation of a signal noise estimate obtained as in [33]. Clusters with an SNR below 1.7 were considered unreliable due to the limitations of the classification algorithm [36].

Analysis of the LFP signals recorded with the large electrodes has also been performed using the Chronux package [37]. One 

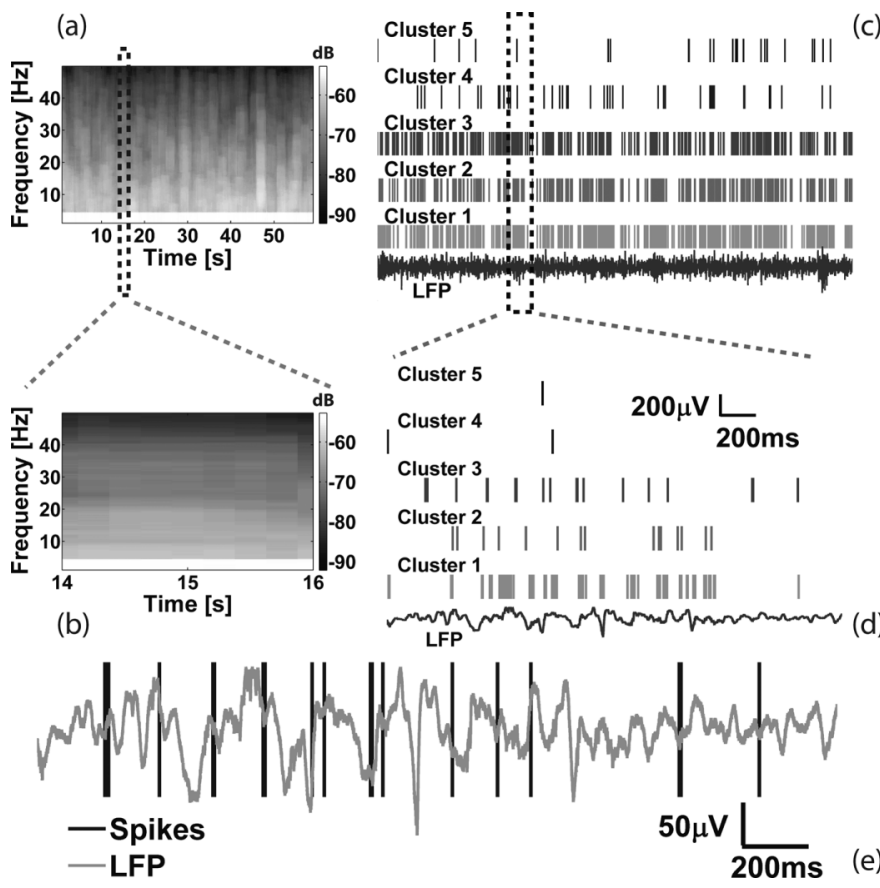

(c)

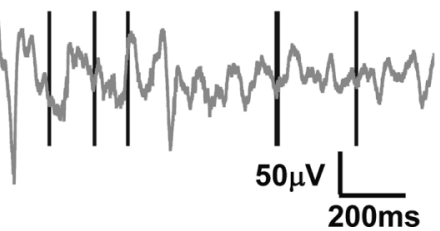

showed coupling to specific phases of the ongoing rhythmic activity (LFP) in different frequency bands. This is demonstrated in Fig. 13(e), where the LFP signal and the activity in Cluster 3 are superimposed. These results demonstrate the relationship between APs and LFPs recorded with the same electrode contact.

\section{CONCLUSION}

We have presented a 16-channel neural recording system with individual channel programmability, which can be used under in vitro and in vivo conditions. The chip is fabricated in a $0.35 \mu \mathrm{m}$ CMOS technology and occupies a total area of $25.2 \mathrm{~mm}^{2}$. This low-noise neural interface is able to accurately separate LFP signals from AP signals, which is useful for real-time visual inspection during in vivo experiments. In vitro experimental results showed successful simultaneous recordings of cardiac-cell signals, with satisfactory signal-to-noise ratios. By applying a simultaneous calcium imaging technique, we were able to demonstrate the correspondence between the recorded spike signals and the calcium transients during spontaneous beating. As the electronic circuits marginally contribute to the overall system noise, we were able to detect APs as small as $40 \mu \mathrm{V}_{p-p}$. Successful in vivo experiments in anesthetized rats have further validated the performance of the recording interface. These experiments, carried out by using micro-machined neural probes with different electrode sizes, showed satisfactory signal-to-noise ratios in different brain regions. We have performed detection and clustering of spontaneous action potentials recorded on each electrode in the neural probes. When applying the spike sorting algorithm to datasets recorded with different probe types (i.e., different electrode sizes), several clusters (i.e., individual neurons) were accurately recognized and separated. Extra analysis of the recorded LFP signals has demonstrated the applicability of the presented neural interface to the study of local synaptic network activity in populations of neurons near the recording electrodes.

\section{ACKNOWLEDGMENT}

The authors would like to thank S. Musa for providing fabrication and packaging of the neural probes and test chips.

\section{REFERENCES}

[1] F. A. Mussa-Ivaldi and L. E. Miller, "Brain-machine interfaces: Computational demands and clinical needs meet basic neuroscience," Trends Neurosci., vol. 26, no. 6, pp. 329-334, Jun. 2003.

[2] K. D. Wise, "Silicon microsystems for neuroscience and neural prostheses," IEEE Eng. Med. Biol. Mag., vol. 24, no. 5, pp. 22-29, 2005.

[3] C. R. Butson and C. C. McIntyre, "Role of electrode design on the volume of tissue activated during deep brain stimulation," J. Neural Eng., vol. 3, no. 1, pp. 1-8, Mar. 2006.

[4] N. K. Logothetis, "The neural basis of the blood-oxygen-level-dependent functional magnetic resonance imaging signal," Philos. Trans. Roy. Soc. London B, Biol. Sci., vol. 357, no. 1424, pp. 1003-1037, 2002.

[5] U. Mitzdorf, "Current source-density method and application in cat cerebral cortex: Investigation of evoked potentials and eeg phenomena," Phys. Rev., vol. 65, no. 1, pp. 37-100, Jan. 1985.

[6] M. A. Montemurro, M. J. Rasch, Y. Murayama, N. K. Logothetis, and S. Panzeri, "Phase-of-firing coding of natural visual stimuli in primary visual cortex," Curr. Biol., vol. 18, no. 5, pp. 375-380, Mar. 2008.

[7] E. J. Hwang and R. A. Andersen, "Effects of visual stimulation on LFPs, spikes, and LFP-spike relations in PRR," J. Neurophys., vol. 105, no. 4, pp. 1850-1860, Apr. 2011. 
[8] G. Kreiman, C. P. Hung, A. Kraskov, R. Q. Quiroga, T. Poggio, and J. J. DiCarlo, "Object selectivity of local field potentials and spikes in the macaque inferior temporal cortex," Neuron, vol. 49, no. 3, pp. 433-445, Feb. 2006.

[9] G. Santhanam, M. D. Linderman, V. Gilja, A. Afshar, S. I. Ryu, T. Meng, and K. Shenoy, "HermesB: A continuous neural recording system for freely behaving primates," IEEE Trans. Biomed. Eng., vol. 54, no. 11, pp. 2037-2050, 2007.

[10] X. Ye, P. Wang, J. Liu, S. Zhang, J. Jiang, Q. Wang, W. Chen, and $\mathrm{X}$. Zheng, "A portable telemetry system for brain stimulation and neuronal activity recording in freely behaving small animals," J. Neurosci. Methods, vol. 174, no. 2, pp. 186-193, Sep. 2008.

[11] M. Chae, K. Chen, W. Liu, J. Kim, and M. Sivaprakasam, "A 4-channel wearable wireless neural recording system," in Proc. IEEE Int. Symp. Circuits and Systems, 2008, pp. 1760-1763.

[12] K. Najafi and K. D. Wise, "An implantable multielectrode array with on-chip signal processing," IEEE J. Solid-State Circuits, vol. 21, no. 6, pp. 1035-1044, 1986.

[13] E. Peeters, B. Puers, W. Sansen, J. Gybels, and P. d. Sutter, "A twowire, digital output multichannel microprobe for recording single-unit neural activity," Sens. Actuators B, Chem., vol. 4, no. 1-2, pp. 217-223, 1991.

[14] R. R. Harrison, P. T. Watkins, R. J. Kier, R. O. Lovejoy, D. J. Black, B. Greger, and F. Solzbacher, "A low-power integrated circuit for a wireless 100-electrode neural recording system," IEEE J. Solid-State Circuits, vol. 42, no. 1, pp. 123-133, 2007.

[15] B. Gosselin, A. E. Ayoub, J.-F. Roy, M. Sawan, F. Lepore, A. Chaudhuri, and D. Guitton, "A mixed-signal multichip neural recording interface with bandwidth reduction," IEEE Trans. Biomed. Circuits Syst., vol. 3, no. 3, pp. 129-141, 2009.

[16] M. S. Chae, Z. Yang, M. R. Yuce, L. Hoang, and W. Liu, "A 128channel $6 \mathrm{mw}$ wireless neural recording IC with spike feature extraction and UWB transmitter," IEEE Trans. Neural Syst. Rehabil. Eng., vol. 17, no. 4, pp. 312-321, 2009.

[17] J. N. Y. Aziz, K. Abdelhalim, R. Shulyzki, R. Genov, B. L. Bardakjian, M. Derchansky, D. Serletis, and P. L. Carlen, "256-channel neural recording and delta compression microsystem with 3D electrodes," IEEE J. Solid-State Circuits, vol. 44, no. 3, pp. 995-1005, 2009.

[18] S. B. Lee, H.-M. Lee, M. Kiani, U.-M. Jow, and M. Ghovanloo, "An inductively powered scalable 32-channel wireless neural recording system-on-a-chip for neuroscience applications," IEEE Trans. Biomed. Circuits Syst., vol. 4, no. 6, pp. 360-371, 2010.

[19] A. C. Hoogerwerf and K. D. Wise, "A three-dimensional microelectrode array for chronic neural recording," IEEE Trans. Biomed. Eng., vol. 41, no. 12, pp. 1136-1146, 1994

[20] R. R. Harrison, G. Santhanam, and K. V. Shenoy, "Local field potential measurement with low-power analog integrated circuit," in Proc. 26th Annu. Int. Conf. IEEE Engineering in Medicine and Biology Society, 2004, vol. 2, pp. 4067-4070.

[21] B. Gosselin, "Recent advances in neural recording microsystems," Sensors, vol. 11, no. 5, pp. 4572-4597, 2011.

[22] D. Hernandez-Garduno and J. Silva-Martinez, "Continuous-time common-mode feedback for high-speed switched-capacitor networks," IEEE J. Solid-State Circuits, vol. 40, no. 8, pp. 1610-1617, 2005.

[23] Z. Yang, Q. Zhao, E. Keefer, and W. Liu, Y. Bengio, D. Schuurmans, J. Lafferty, C. K. I. Williams, and A. Culotta, Eds., "Noise characterization, modeling, and reduction for in vivo neural recording," in Proc. Advances in Neural Information Processing Systems 22, 2009, pp. $2160-2168$.

[24] X. Liu, A. Demosthenous, and N. Donaldson, "Platinum electrode noise in the ENG spectrum," Med. Biol. Eng. Comput., vol. 46, no. 10, pp. 997-1003, Oct. 2008.

[25] S. F. Lempka, "The Electrode-Tissue Interface During Recording and Stimulation in the Central Nervous System," Ph.D. dissertation, Case Western Reserve Univ., Cleveland, OH, 2010.

[26] W. Wattanapanitch, M. Fee, and R. Sarpeshkar, "An energy-efficient micropower neural recording amplifier," IEEE Trans. Biomed. Circuits Syst., vol. 1, no. 2, pp. 136-147, 2007.

[27] M. S. J. Steyaert and W. M. C. Sansen, "A micropower low-noise monolithic instrumentation amplifier for medical purposes," IEEE $J$. Solid-State Circuits, vol. 22, no. 6, pp. 1163-1168, 1987.

[28] R. R. Harrison and C. Charles, "A low-power low-noise CMOS amplifier for neural recording applications," IEEE J. Solid-State Circuits, vol. 38 , no. 6 , pp. 958-965, 2003.
[29] R. S. Assaad and J. Silva-Martinez, "The recycling folded cascode: A general enhancement of the folded cascode amplifier," IEEE J. SolidState Circuits, vol. 44, no. 9, pp. 2535-2542, Sep. 2009.

[30] M. Yin and M. Ghovanloo, "A low-noise preamplifier with adjustable gain and bandwidth for biopotential recording applications," in Proc. IEEE Int. Symp. Circuits and Systems, 2007, pp. 321-324.

[31] S. Musa, M. Welkenhuysen, R. Huys, W. Eberle, K. Kuyck, C. Bartic, B. Nuttin, and G. Borghs, "Planar 2D-array neural probe for deep brain stimulation and recording (DBSR)," in Proc. 4th Eur. Conf. Int. Federation for Medical and Biological Engineering, Berlin, Germany, 2009, vol. 22, pp. 2421-2425.

[32] C. H. Luo and Y. Rudy, "A dynamic model of the cardiac ventricular action potential. I. Simulations of ionic currents and concentration changes," Circ. Res., vol. 74, no. 6, pp. 1071-1096, Jun. 1994.

[33] R. Q. Quiroga, Z. Nadasdy, and Y. Ben-Shaul, "Unsupervised spike detection and sorting with wavelets and superparamagnetic clustering," Neural Comput., vol. 16, no. 8, pp. 1661-1687, 2004.

[34] M. Blatt, S. Wiseman, and E. Domany, "Superparamagnetic clustering of data," Phys. Rev. Lett., vol. 76, no. 18, pp. 3251-3254, Apr. 1996.

[35] U. Rutishauser, E. M. Schuman, and A. N. Mamelak, "Online detection and sorting of extracellularly recorded action potentials in human medial temporal lobe recordings, in vivo," J. Neurosci. Methods, vol. 154, no. 1-2, pp. 204-224, Jun. 2006.

[36] I. Gligorijevic, M. Welkenhuysen, D. Prodanov, W. Eberle, B. Nuttin, C. Bartic, and S. V. Huffel, "Statistical analysis of neural spike trains for evaluation of functional differences in brain activity," in Proc. Biosignal Conf., 2010.

[37] P. Mitra and H. Bokil, Observed Brain Dynamics Oxford Univ. Press, New York, 2007 [Online]. Available: http://chronux.org

[38] C. K. Young and J. J. Eggermont, "Coupling of mesoscopic brain oscillations: Recent advances in analytical and theoretical perspectives," Prog. Neurobiol., vol. 89, no. 1, pp. 61-78, Sep. 2009.

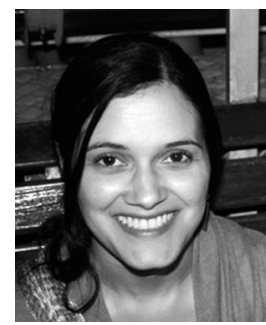

Carolina Mora Lopez (S'10) received the B.S. degree in electrical engineering from Universidad de Antioquia, Medellin, Colombia, in 2004, and the M.S. degree in electrical engineering from the Polytechnic University of Catalonia, Barcelona, Spain, in 2007.

Currently, she is working toward the Ph.D. degree in microelectronic interfaces for biomedical applications at Imec, Leuven, Belgium, and Katholieke Universiteit Leuven, Belgium. Her research interests include analog and mixed-signal circuit design for sensor and bioelectronic interfaces.

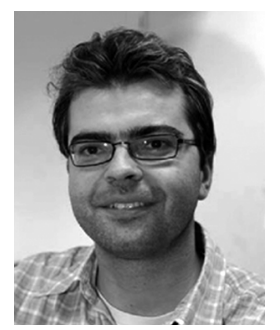

Dimiter Prodanov was born in Sofia, Bulgaria, on March 16, 1975. He graduated the National High School of Mathematics and Sciences, Sofia, Bulgaria, in 1993 with a degree summa cum laude, and received the MD degree from the Medical University Sofia, Bulgaria, in 1999, and the Ph.D. degree in neuroscience from Twente University, Enschede, The Netherlands, in 2006.

He was awarded a John G. Nicholls Fellowship from the International Brain Research Organization (IBRO) in 2006 to continue research in neuroscience at the Catholic University of Louvain, Belgium. In 2008, he completed postdoctoral training at the University of Liege, Belgium. Since 2008, he has been a Senior Biomedical Scientist at the Nanoelectronics Research Center Imec, Leuven, Belgium, and Associate to the Laboratory for Experimental Functional Neurosurgery, Catholic University of Leuven, Belgium. His research interests include selective electrical stimulation and recording of the brain activity, development of brain-computer interfaces, digital microscopy and biomedical imaging, neurophysiological signal processing and image analysis.

Dr. Prodanov is a member of IBRO, the Belgian Neurological Society, the Belgian Society for Neuroscience, editorial board member of Head and Face Medicine, and the scientific advisory board to the ImageJ User and Developer Conference. 


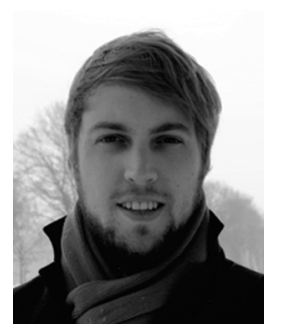

Dries Braeken received the M.Sc. degree in biomedical sciences from Katholieke Universiteit Leuven, Belgium, in 2003, and the Ph.D. degree in medical sciences from the Katholieke Universiteit Leuven (in collaboration with Imec, Leuven, Belgium) in 2009.

Since 2009, he has been a Senior Researcher in the Biolectronic Systems group at Imec in the field of in vitro biomedical research. He is member of the Society for Neuroscience (SfN) and the Engineering in Medicine and Biology Society (EMBS). He is (co)author of more than 30 internationally peer-reviewed publications. His research interests are in the development and validation of advanced electrophysiological tools for cardio- and neurophysiological research.

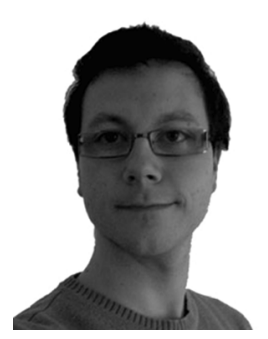

Ivan Gligorijevic received the degree of electrical engineering in the Faculty of Electrical Engineering, University of Belgrade, Serbia, in 2008 after completing a master's thesis in optical sensors at Katholieke Universiteit Leuven, Belgium.

In 2009, he became a Ph.D. candidate in the Biomed group, Department of Electrical Engineering (ESAT-SCD/SISTA), K.U. Leuven, Belgium, under supervision of Prof. Sabine Van Huffel. The primary research area is connected to developing signal processing solutions for closing the loop between deep brain stimulation (DBS) and recording for microarray probes in cooperation with Imec, Leuven, Belgium.

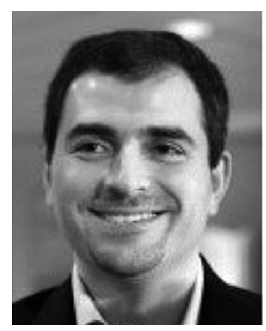

Wolfgang Eberle (M'00-SM'07) received the M.Sc. degree in electrical engineering from Saarland University, Saarbrucken, Germany, in 1996, with thesis projects on transceiver design for wireless body area networks and transcutaneous energy and data transmission. In 2006, he received the Ph.D. degree in electrical engineering from Katholieke Universiteit Leuven, Belgium, with a dissertation on mixed analog/digital exploration and design for wireless broadband transceivers.

He joined Imec in 1997 as a Wireless Systems Engineer. He consecutively had functions as a Wireless VLSI Engineer, Senior Wireless Engineer, Team Leader Mixed-Signal CAD, and Activity Leader Wireless Multimedia Systems. In 2006, he joined Imec's biomedical research deparment as a Project Manager/Senior Scientist. Since 2009, he has been an R\&D Manager of the Bioelectronic Systems Group and Program Manager Cell interfacing technology. He was a Consultant to startups Cardiomed $\mathrm{GmbH}$ in 1993 and Resonext Communications Inc. in 2002. He is (co-)inventor of eight patents and (co-)author of more than 70 scientific publications including one book edited by Springer.

Dr. Eberle is the Conferences and Events Officer of the IEEE Engineering in Medicine and Biology Society Benelux Chapter. He has been a regular committee member and topic chair for Design, Automation and Test in Europe and a regular reviewer for biomedical, electronics, and wireless conferences and journals.

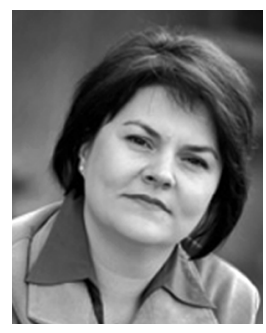

Carmen Bartic received the Ph.D. degree in physics from the Katholieke Universiteit Leuven, Belgium, in 2002 with a thesis on organic-based field-effect transducers for bioanalytical applications (the first $\mathrm{pH}$ and glucose OFET sensors).

Since 2002, she has been with Imec, Leuven, Belgium, in different positions, including Senior Scientist, Group Leader, and Program Manager. During the period 2003-2009, she coordinated the research activities of the Bioelectronic Systems group of Imec, working on neuro-electronic inter- facing technologies. She has been one of the initiators of the Neuroelectronics Research Center of Flanders (NERF), a multidisciplinary collaborative institute created by K.U. Leuven, Flemish Institute for Biotechnologies (VIB) and Imec. In October 2009, she joined the Department of Physics and Astronomy of the K.U. Leuven as Associate Professor on Nanobiophysics. Her research focuses on functional interactions between nano and microfabricated systems and cells (e.g., nanosensors, techniques to probe cellular functions at nanoscale). She has extensive expertise in neuro-electronic hybrid systems, biosensor design and fabrication technologies, and technologies for on chip cellular microenvironments.

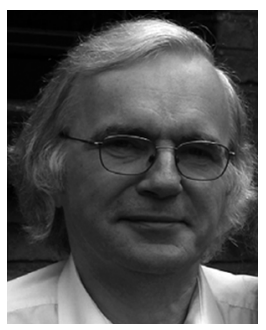

Robert Puers (M'86-SM'95-F'11) received the Ph.D. degree from Katholieke Universiteit Leuven (K.U. Leuven), Leuven, Belgium, in 1986.

At K.U Leuven, he became Director of the Clean Room Facilities for Silicon and Hybrid Circuit Technology at the ESAT-MICAS laboratories. $\mathrm{He}$ was a European pioneer in the research on micromachining, MEMS and packaging techniques, mainly for biomedical implantable systems. To this purpose, he assembled the requested infrastructure, and installed a clean room in 1984, that now runs for more than 25 years under his guidance. Recently, microfluidic and optical MEMS based on polymers are forming the backbone of his sensor research Beside MEMS, his work focuses also on low power systems, smart interfaces, inductive power and wireless communication. He published design guidelines to improve the efficiency of power induction in two books. He took major efforts to increase the impact of MEMS and microsystems in both the international research community as well as in industry. He helped to launch two spin-off companies.

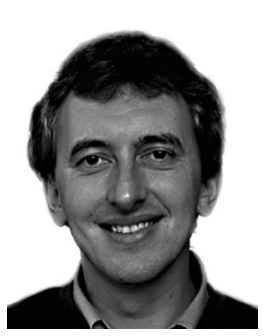

Georges Gielen (S'87-M'92-SM'99-F'02) received the M.S. and Ph.D. degrees in electrical engineering from Katholieke Universiteit Leuven, Leuven, Belgium, in 1986 and 1990, respectively.

In 1990, he was a Post-Doctoral Research Assistant and Visiting Lecturer with the Department of Electrical Engineering and Computer Science, University of California, Berkeley. From 1991 to 1993, he was a Post-Doctoral Research Assistant with the Belgian National Fund of Scientific Research, ESAT Laboratory, Katholieke Universiteit Leuven. In 1993, he was an Assistant Professor with Katholieke Universiteit Leuven, where he was promoted to Full Professor in 2000. He has authored or co-authored two books and more than 300 papers in edited books, international journals, and conference proceedings. His current research interests include the design of analog and mixed-signal integrated circuits, and especially analog and mixed-signal computer-aided design tools and design automation (modeling, simulation and symbolic analysis, analog synthesis, analog layout generation, analog, and mixed-signal testing). He is a coordinator or a partner of several (industrial) research projects in this area.

Dr. Gielen is regularly a member of the program committees of international conferences (DAC, ICCAD, ISCAS, DATE, CICC, and so on), and served as the General Chair of the DATE Conference in 2006 and of the ICCAD Conference in 2007. He serves regularly as a member of editorial boards of international journals (IEEE TRANSACTIONS ON CIRCUITS AND SYSTEMS, Springer International Journal on Analog Integrated Circuits and Signal Processing, Elsevier Integration). He received the 1995 Best Paper Award in the John Wiley International Journal on Circuit Theory and Applications, and was the 1997 Laureate of the Belgian Royal Academy on Sciences, Literature and Arts in the Discipline of Engineering. He received the 2000 Alcatel Award from the Belgian National Fund of Scientific Research for his innovative research in telecommunications, and won the DATE 2004 Best Paper Award. He served as an elected member of the Board of Governors of the IEEE Circuits and Systems (CAS) Society and as the Chairman of the IEEE Benelux CAS Chapter. He served as the President of the CAS Society in 2005. 